\title{
DISTRIBUTED NEUROCHEMICAL SENSING: IN VITRO EXPERIMENTS
}

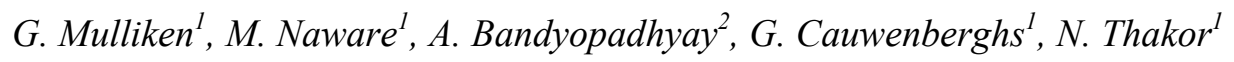 \\ ${ }^{1}$ BME Dept. Johns Hopkins University School of Medicine, Baltimore, MD 21205 \\ ${ }^{2}$ ECE Dept. Johns Hopkins University, Baltimore, MD 21218
}

\begin{abstract}
Experimental results characterizing a VLSI multi-channel potentiostat sensor system designed for sensing distributed neurotransmitter activity are presented. Neurotransmitter concentration is electrochemically transduced using an external carbon fiber electrode. Resultant current is processed by an integrated potentiostat, consisting of a current amplification stage, current-mode delta-sigma A/D converter, and counting decimator. Electrical characterization has shown that the VLSI potentiostat is sensitive to picoampere levels of input current. Furthermore, both static and dynamic neurochemical measurements of dopamine are verified in vitro, proving the utility of the device for brain slice studies. Lastly, a biologically inspired experiment, whereby the catabolism of dopamine is emulated with the addition of the catechol-o-methyl transferase (COMT) enzyme to modulate dopamine levels in vitro.
\end{abstract}

\section{INTRODUCTION}

Understanding the onset and progression of neurological injury or its pathological response demands a detailed knowledge of the neurotransmitter mechanisms underlying these processes. While advances in electrophysiology have borne significant insights into how the brain processes information 'electrically', far less progress has been made in mapping the concurrent, chemical manifests of neural activity. By quantifying the spatiotemporal concentration of neurotransmitters in real time, distributed neurochemical sensors will provide an enhanced perspective from which to study the role of neurotransmitters in brain injury and its sequelae.

The ability to electrochemically sense neurotransmitter activity from multiple electrodes carries with it a formidable instrumentation challenge, demanding the implementation of numerous potentiostats in parallel. Use of multiple benchtop potentiostats offers a crude and impractical solution, consuming significant area on the laboratory bench, dissipating large amounts of power, and offering no flexibility for in vivo experimentation. Alternatively, a highly integrated VLSI chip can provide the same processing capability on a single substrate less than $2 \mathrm{~mm}^{2}$ in area [1]. Furthermore, a VLSI implementation will drastically reduce power consumption, decrease manufacturing costs, and minimize noise associated with unnecessary wiring. A few attempts have been made to develop VLSI potentiostats. However, in all cases, the designs have included only a single channel [2-4]. Lastly, future innovative sensor systems can be realized to target in vivo applications, combining sensors and microelectronics onto a single substrate and wirelessly transmitting neurochemical information for remote processing.

\section{SYSTEM ARCHITECTURE}

The VLSI potentiostat operates in a two-electrode configuration, which is sufficient for neurotransmitter measurements since the voltage drop (IR) due to fluid resistance is negligible for the very small current levels being sensed. That is, a counter electrode to eliminate current flow into the reference electrode is obviated since the current levels are so small [5]. Each channel employs a working electrode, which is maintained at virtual ground. Additionally, a single reference electrode is biased at $-\mathbf{E}_{\text {redox }}$ with respect to the working electrodes. Thus the redox potential of the neurotransmitter being oxidized is maintained between all working electrodes and the reference electrode.

\subsection{Chip Architecture}

Each potentiostat channel consists of a current amplification stage, with input current conveyor and programmable gain current mirrors, and a current-mode delta-sigma A/D converter, with counting decimator. A register buffer and shift register provide an asynchronous bit-serial output. The potentiostat architecture is shown in Figure 1. The chip operates in the subthreshold region to provide high sensitivity for the wide range of expected input currents and to decrease power consumption. Circuit implementation details are presented in [1].

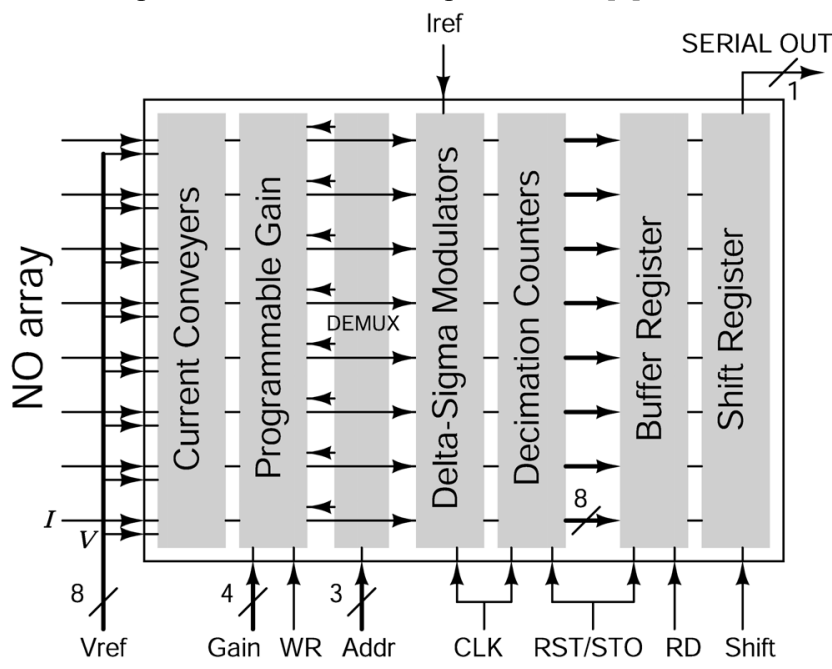

Figure 1. Potentiostat chip architecture [1].

Electrochemical measurements of neurotransmitter generally transduce currents on the order of picoamperes to nanoamperes $[4,6]$. Input current in each channel is amplified to the microampere range and passed to a delta-sigma $\mathrm{A} / \mathrm{D}$ converter to digitize the signal. The oversampled binary output of the deltasigma modulator is decimated using a binary counter. The 
decimated signals for all channels are then scanned through an output shift register, whereby the sequence is fed off chip through an asynchronous bit-serial interface for external processing.

A $1 \mathrm{MHz}$ clock (CLK) serves as the system clock for the deltasigma modulators and decimation counters. One sample of conversion takes 4,096 cycles of the system clock to obtain 12bit resolution at $\sim 250 \mathrm{~Hz}$ conversion rate. Neurotransmitter activity operates at low frequency when compared to most digital data-processing rates. For instance, the synaptic delay of chemical synapses, including the arrival of the presynaptic action potential, exocytosis and binding of neurotransmitter to the postsynaptic membrane, can last around milliseconds [7]. Furthermore, nitric oxide, which is known for being a short-lived neurotransmitter, has a half-life on the order of seconds [8]. Delta sigma modulation is well suited for such low-bandwidth analog inputs.

\subsection{In Vitro Potentiostat Data Acquisition System}

A data acquisition system supports the potentiostat chip to enable real-time in vitro measurements in the laboratory. Serial data is read asynchronously into personal computer (PC) memory through a data acquisition card, while power, biases, and synchronous input clocks are provided from a stand-alone electronics board. Data acquisition code is written in $\mathrm{C}^{++}$and is supported by Virtual Library® drivers.

\section{RESULTS}

\subsection{Electrical Characterization of Potentiostat}

The potentiostat chip is fabricated in $0.5 \mu \mathrm{m}$ technology, with an active area of $1.1 \mathrm{~mm} \times 1.1 \mathrm{~mm}$. Power consumption is $0.5 \mathrm{~mW}$ at $5 \mathrm{~V}$ supply, and 8-channel operation.

\subsubsection{Current Amplification Verification}

For verification purposes, bi-directional output current from the current gain stage is made accessible in the chip design through a pair of test pins. Physiological levels of current are swept in a ramp-like fashion and current outputs are acquired and digitized, yielding a transfer curve for the input gain stage. Proper amplification is verified for various gain settings.

Results show that the potentiostat input stage is sensitive to levels of current ranging from picoamperes (pA) to microamperes $(\mu \mathrm{A})$, sufficient for measurement of neurotransmitter under physiological conditions [1]. Note that gain mismatch is a result of the positive and negative currents being conveyed through two different current mirror paths. These slope differences can be accounted for by knowing the polarity and gain setting of the channel, and calibration in software. Additionally, any offset resulting from transistor mismatch in the differential amplifier of the input stage conveyor is calibrated through accurate control of the reference voltage $\mathbf{V}_{\text {ref }}$ (the potential applied to the working electrode).

\subsubsection{Delta Sigma A/D Converter Verification}

The delta sigma A/D is first characterized for 8-bit conversion, at unity current gain. To obtain a calibration curve of digital output versus current input, current is ramped from the negative to positive saturation limits as before. A single 8-bit word is read asynchronously following each conversion. A sweep from approximately $-20 \mathrm{nA}$ to $+20 \mathrm{nA}$ is characterized (see Figure 2).

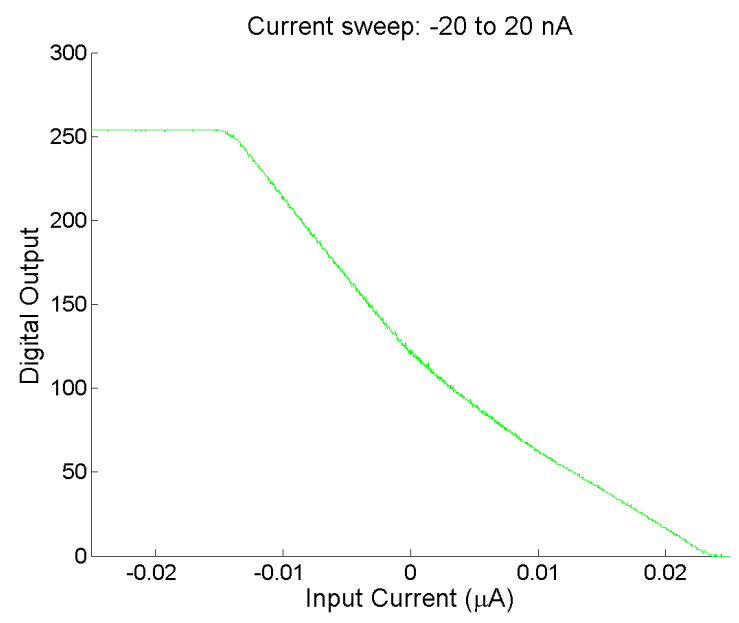

Figure 2. Calibration curve for a single channel's digital output (without averaging).

\subsection{Neurochemical Characterization of Sensing System}

In vitro neurotransmitter measurements are made for dopamine, verifying the utility of the VLSI potentiostat for neuroscience research.

\subsubsection{Carbon Fiber Sensor Preparation and Calibration}

Uncoated carbon fiber electrodes, $30 \mu \mathrm{m}$ in diameter and $250 \mu \mathrm{m}$ in length (World Precision Instruments, USA), were used for all recordings. Electrode activation and analyte calibration is performed using the EG\&G Model 273-A Potentiostat/Galvanostat (Princeton Applied Research, USA) connected to a computer. Electrodes are activated in phosphate buffered saline (PBS) solution ( $\mathrm{pH}=7.4)$ using successive voltage cycling as described by Park et al. [6]. Activation of the electrode increases sensitivity by as much as ten-fold and enhances reversibility of the electrode response, making it less susceptible to background noise [5]. Secondly, calibration curves of concentration versus current are obtained for dopamine using standard chronoamperometric methods.

\subsubsection{VLSI Potentiostat Preparation}

First, a chamber is filled with $100 \mathrm{ml}$ of PBS solution. An $\mathrm{Ag} / \mathrm{AgCl}$ reference electrode (Bioanalytical Systems) and the carbon fiber working electrode are placed into the bath. The working electrode is connected to the potentiostat's inverting input terminal and the reference electrode is biased through a control amplifier in a voltage follower configuration. The working electrode is held at $\mathbf{V}_{\text {ref }}=2.5 \mathrm{~V}$, and the reference voltage is set to $2.15 \mathrm{~V}$ to apply dopamine's redox potential of $350 \mathrm{mV}$. Chronoamperometric measurements are made with the VLSI potentiostat, and real-time digital output is stored to PC 
memory. A simplified schematic of the setup is given in Figure 3.

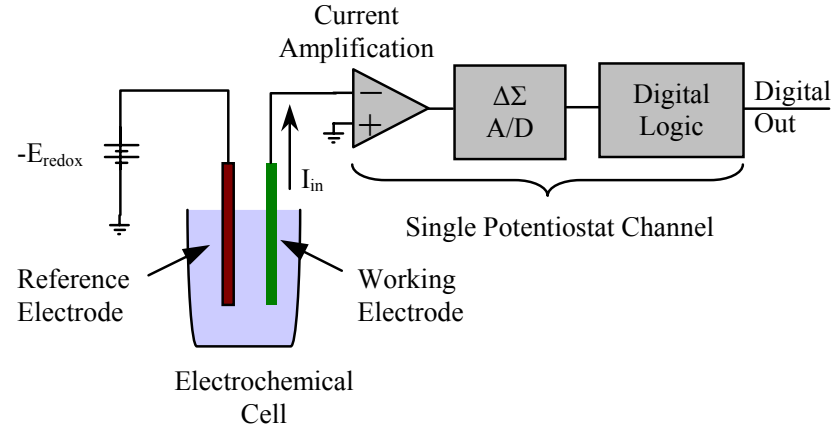

Figure 3. Simplified schematic of potentiostat system and interfacing electrochemical cell.

\subsubsection{Static Dopamine Measurements}

First, a baseline current is obtained for PBS solution, without dopamine. Seven solutions of increasing concentration are prepared one at a time. The concentrations of dopamine in the solutions are 2.5, 5.0, 7.5, 10.0, 15.0, 20.0 and $30.0 \mu \mathrm{g} / \mathrm{ml}$. The sensor is placed into each solution and measurements are made after 2 minutes. Steady-state digital outputs are compiled from single measurements to generate a static calibration curve, shown below in Figure 4.

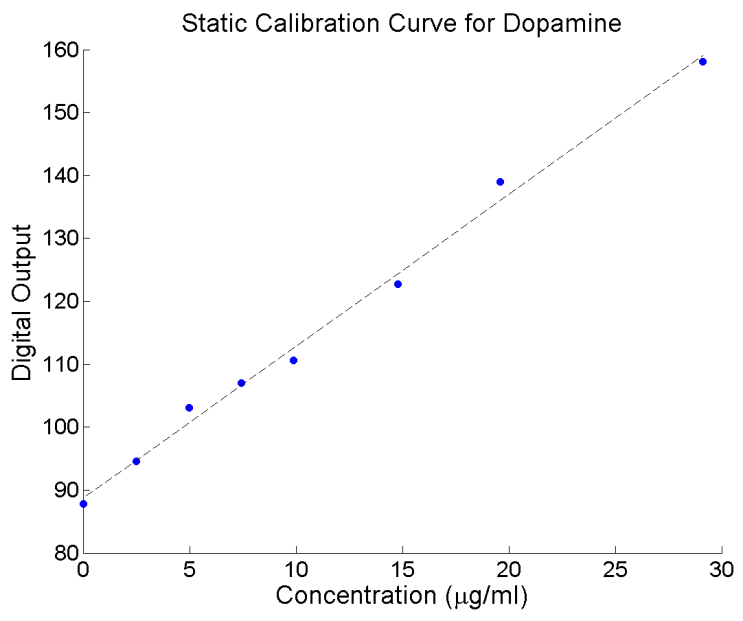

Figure 4. Static calibration curve for dopamine. Starting from baseline $(0 \mu \mathrm{g} / \mathrm{ml})$, single, steady-state measurements are made for seven solutions of increasing concentration.

Ambient noise due to changing experimental conditions, minor inconsistencies in measured volumes and degradation of the dopamine standard solution itself (degrades in $\sim 1$ hour) may account for the deviations from linearity.

\subsubsection{Dynamic Dopamine Measurements}

Again, a baseline current is obtained for PBS solution. A $500 \mu \mathrm{l}$ gastight syringe (Harvard Apparatus) is used to deliver dopamine into the PBS bath. Specifically, $500 \mu$ incremental boluses of a
$1 \mathrm{mg} / \mathrm{ml}$ dopamine standard solution are added to create step increases in digital output, offset in time by approximately five minutes (see Figure 5).

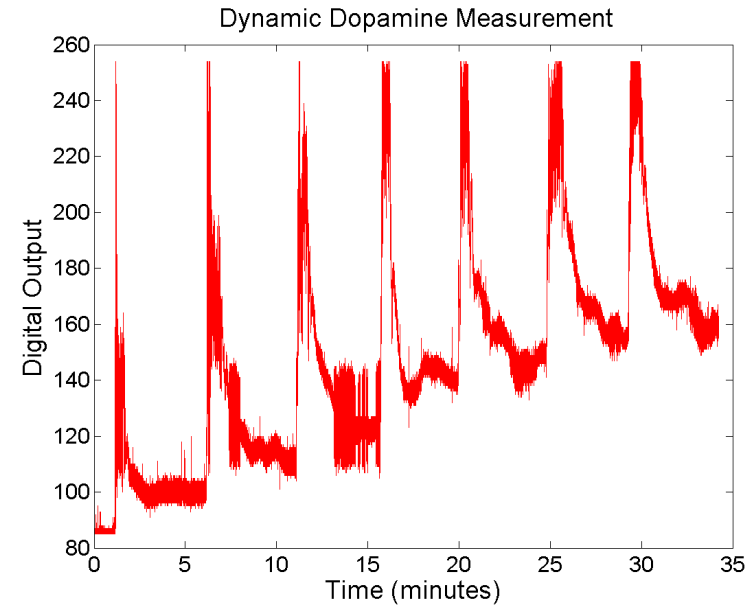

Figure 5. Dynamic calibration curve obtained by continuous potentiostat measurement during $500 \mu \mathrm{l}$ step increases of dopamine, infused every $\sim 5$ minutes (single measurement).

Large transients at the beginning of each step increase are due to infusion and subsequent stirring to ensure uniform dilution of dopamine. Increased settling time for larger concentrations is likely due to the inherent limitations of the two-electrode configuration, whereby larger IR drops between reference and working electrodes may result in instability. To obtain a cleaner plot, steady-state outputs are averaged for each step and then plotted again versus time as seen below in Figure 6.

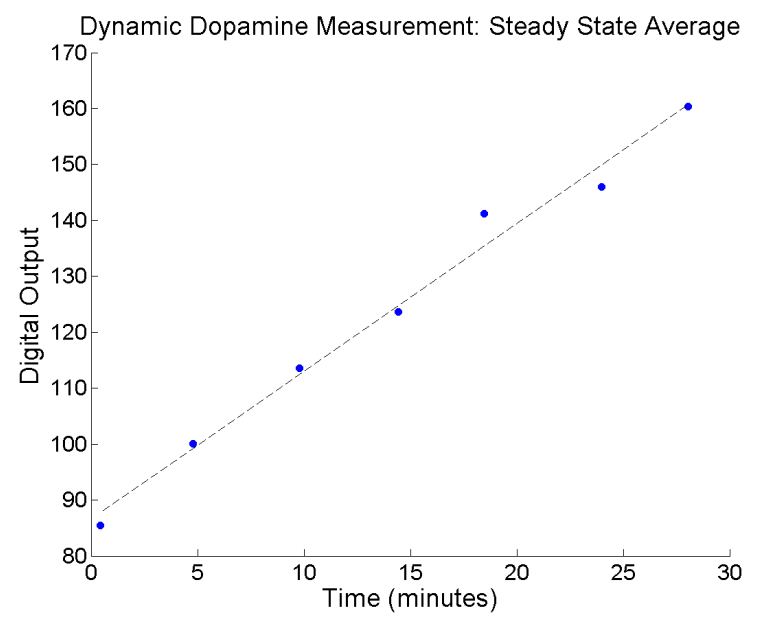

Figure 6. Dynamic calibration curve for dopamine obtained after averaging steady-state output following each infusion, from Figure 5.

\subsubsection{Biological Modulation of Dopamine In Vitro}

Catechol-o-methyl transferase (COMT), exists in the extracellular space and catabolizes dopamine to form the metabolite 3-methoxytyramine [9]. Interestingly, inhibitors of 
COMT (and its intracellular counterpart, monoamine oxidase) have been used in the treatment of Parkinson's disease to increase dopamine levels [9]. In vitro catabolism of dopamine can be accomplished by introducing COMT to dopamine solution in vitro.

The COMT assay is prepared by mixing the following reagents in the amounts indicated:

1. The cofactor, S-Adenosylmethionine (SAM), $(0.435$ $\mathrm{mg} / \mathrm{ml}$ ) $100 \mathrm{ml}$. (This product must be of highest purity and must not contain traces of Sadenosylhomocysteine).

2. $10 \mathrm{mM}$ Magnesium chloride, in distilled water $(0.952$ $\mathrm{mg} / \mathrm{ml}) .100 \mathrm{ml}$.

3. $0.5 \mathrm{M}$ Tris- $\mathrm{HCl}$ buffer, $\mathrm{pH} 8.0$ in distilled water. $100 \mathrm{ml}$.

4. COMT (enzyme) solution: Prepared a suitable dilution (2:1, enzyme:cofactor) of the enzyme using cold distilled water. Prepared fresh prior to assay.

First, baseline measurements for de-ionized water and a constantconcentration dopamine solution $(2 \mathrm{mg} / \mathrm{ml})$ in deionized water are measured, yielding digital outputs of 98 and 105 respectively. Constant-concentration dopamine solution and assay solution are

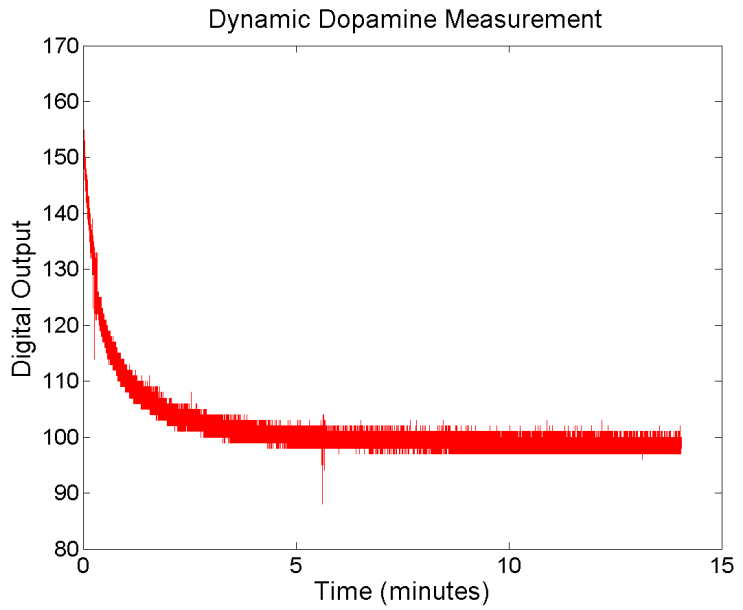

Figure 7. Dynamic measurement of dopamine catabolism by COMT enzyme.

next incubated for 15 minutes at $37^{\circ} \mathrm{C} .4 \mathrm{ml}$ of the COMT assay solution is then pipetted into the beaker and chronoamperometric measurements are made for a period of fifteen minutes with the expectation that the level of dopamine will decrease as it is degraded by COMT. Indeed, results show an exponential decrease in dopamine concentration over time as seen above in Figure 7 . The steady state value reaches 98 after approximately 8 minutes (the calibrated zero current value for de-ionized water), when all of the dopamine has been catabolized.

\section{SUMMARY}

In vitro experiments using a multi-channel VLSI potentiostat system for distributed neurochemical recording are presented. A single channel is characterized electrically and electrochemically, using a test assay of the neurotransmitter dopamine. The VLSI potentiostat is the first to integrate multiple potentiostats onto a single chip and promises to serve as a valuable tool for neurophysiological research. The device employs current-mode analog circuitry and operates in the subthreshold domain to increase sensitivity and decrease power consumption. The VLSI potentiostat chip acquires eight input currents and processes them at a sampling rate of $250 \mathrm{~Hz}$, outputting all eight channels' output in serial format. In vitro experimentation is supported by a custom data acquisition system that provides power, biases and clocks to the chip as well as an asynchronous data link to a PC. Lastly, telemetry development has already begun and further testing of a wireless potentiostat in vitro is a logical next step in the sensor development. An implantable, multi-channel neurochemical sensor will enable numerous experimental protocols for basic research and open new possibilities for clinical applications.

\section{REFERENCES}

[1] Bandyopadhyay A, Mulliken G, Cauwenberghs G, and Thakor N., VLSI Potentiostat Array for Distributed Electrochemical Neural Recording. IEEE International Symposium on Circuits and Systems, 2002. ISCAS 2002, 2002. 2: p. $740-743$.

[2] Kakerow RG, Kappert H, Spiegel E, Manoli Y, Low-power single-chip CMOS potentiostat. Transducers - Eurosensors IX, 1995. 1: p. 142-145.

[3] Reay RJ, K.S., Kovacs GTA, An integrated CMOS Potentiostat for Miniaturized Electroanalytical Instrumentation. IEEE International Solid-State Circuits Conference, 1994. 1: p. 162-163.

[4] Turner RBF, H.D., Baltes HP, A CMOS potentiostat for amperometric chemical sensors. IEEE Solid-State Circuits, 1987. SC-22: p. 473-478.

[5] World Precision Instruments, Application Note: Carbon fiber and carbon disk microelectrodes for electrochemical analysis and electrophysiological recording, 2002:

[6] Park JK, T.P., Chao JKT, Ghodadra R, Rangarajan R, Thakor NV, In vivo nitric oxide sensor using nonconducting polymer-modified carbon fiber. Biosensors \& Bioelectronics, 1998. 13: p. 1187-1195.

[7] Kandel ER, S.S., Overview of Synpatic Transmission, in Principles of Neural Science, S.J. Kandel ER, Jessell TM, Editor. 2000, McGraw-Hill Companies: USA.

[8] Vallance P, P.S., Bhagat K, MacAllister R, Radomski M, Moncada S, Malinski T, Direct measurement of nitric oxide in human beings. The Lancet, 1995. 346: p. 153-154.

[9] Elsworth JD, R.R., Dopamine synthesis, uptake, metabolism, and receptors: relevance to gene therapy of Parkinson's disease. Exp Neurol, 1997. 144(1): p. 4-9. 